\title{
Three Components of Individualism
}

\author{
ANU REALO ${ }^{1,2 *}$, KATI KOIDO $^{2}$, EVA CEULEMANS ${ }^{1}$ and JÜRI ALLIK ${ }^{2}$ \\ ${ }^{1}$ University of Leuven, Belgium \\ ${ }^{2}$ University of Tartu, Estonia
}

\begin{abstract}
In this article, following an assumption that individualism and collectivism are separate factors, we have further established that three central components of individualism can be distinguished. In the first part of the article we examined whether the three proposed components of individualism-autonomy, mature self-responsibility, and uniquenesscan be distinguished from each other in one cultural context, Estonia. A new scale was developed to measure the three aspects of individualism which demonstrated both the reasonable internal-consistency reliability as well as convergent and divergent validity with several other measures of individualism and collectivism and related constructs. In the second part of the article we studied whether individualism generalizes across specific contexts or domains of social relationships, namely, across relations with family and close others; friends and peers; state and nation. The results of the three-mode principal component analysis showed that the individualistic tendencies of the respondents did not differ much while measured toward the three types of social relation. Copyright (C) 2002 John Wiley \& Sons, Ltd.
\end{abstract}

\section{INTRODUCTION}

The constructs of individualism and collectivism have received a lot of researchers' attention. While the two concepts have been popular in most of the social sciences for about a century, the more systematic and intensive investigation in psychology began after the influential study of Hofstede (1980), who found that about one-half of the country-tocountry differences could be explained by four dimensions labelled as power distance, uncertainty avoidance, masculinity-femininity and individualism-collectivism. Even though the 1980s were already called 'the decade of individualism-collectivism in crosscultural psychology' (Kagitçibasi, 1994, p. 52), the research on the two constructs continued to flourish through the 1990s and shows only vague signs (if any at all) of calming down at the beginning of the third millennium.

*Correspondence to: Anu Realo, Department of Psychology, University of Leuven, Tiensestraat 102, Leuven 3000, Belgium. E-mail: anu.realo@psy.kuleuven.ac.be

Contract/grant sponsor: Research Council of the University of Leuven.

Contract/grant number: F/00/036; GOA/00/02. 
Since Hofstede's groundbreaking study (1980), the individualism and collectivism constructs have undergone a series of elaborations. In the present age, for instance, individualism and collectivism are conceptualized not as two opposite poles of a unidimensional factor as done by Hofstede (1980) but as two relatively independent factors at both the cultural and individual levels ${ }^{1}$ (Triandis et al., 1986; Triandis, Bontempo, Villareal, Asai, \& Lucca, 1988; Triandis, McCusker, \& Hui, 1990). Also, it has been argued that individualism and collectivism are not completely generalizable and context-free dispositions. According to Triandis (2001), 'there are as many varieties of collectivism as there are collectivist cultures' (p. 909) and individualism and collectivism have various subforms that manifest themselves predominantly in one particular area of social relations or in relations with a specific target group.

Despite the great enthusiasm and advancement, there are still several fundamental issues that need to be solved. As stated by Kagitçibasi (1997), 'much research is [still] needed to determine the limits of the constructs of individualism-collectivism' (p. 28). This and several other calls for refinement in conceptualization and measurement of individualism and collectivism have raised questions about the meaning, dimensionality, basic attributes, and operationalization of the constructs both at the individual and cultural levels (cf. Chen, Meindl, \& Hunt, 1997; Fijneman, Willemsen, \& Poortinga, 1996; Kagitçibasi, 1994, 1997).

This paper concentrates mainly on individualism, following the assumption that individualism and collectivism are not necessarily the opposite poles of the same dimension. In response to calls for conceptual specification of individualism and collectivism, the aim of this study is to refine the meaning of individualism and to examine its structure at the individual level. More specifically, we focus on two related issues in this paper. The first issue concerns the definition of individualism. Namely, what are the core themes of individualism? The second issue concerns the context-specific nature of individualism, specifically, whether individualistic orientations generalize across various domains of social relations. In the next section we shall briefly review what has been said about these two issues in the literature on individualism and collectivism.

\section{Definitions and attributes of individualism}

The first question of this article — what are the defining attributes of individualism? — is nearly as old as the term 'individualism' itself. Being merely a 19th-century expression, the term has a rich semantic history, having been 'used in a great many ways, in many different contexts and with an exceptional lack of precision' (Lukes, 1971, p. 45). According to Lukes (1971), the first uses of the term grew out of the general European reaction to the French Revolution and, more specifically, to its declared source, the thought of Enlightenment. The early ideas of individualism in social and political theory included the ideas of the maximum welfare and freedom of the individual, with society existing only for the sake of its members. More specifically, individualism is a 'political and social philosophy that places high value on the freedom of the individual and generally stresses the self-directed, self-contained, and comparatively unrestrained individual or ego. [...] The individualistic theory of human nature holds that the interests of the normal adult are

\footnotetext{
${ }^{1}$ Although Triandis, Leung, Villareal, and Clack (1985) have proposed using terms individualism and collectivism for analyses at the cultural level and terms idiocentrism and allocentrism for analyses at the individual level, the more popular and widely used concepts individualism and collectivism are employed in this paper to describe both cultural and individual level constructs.
} 
best served by allowing him maximum freedom and responsibility for choosing his objectives and the means for obtaining them, and acting accordingly' (Encyclopaedia Britannica, www.britannica.com).

In psychology, one of the first operationalizations of individualism was offered by Hofstede $(1980,1991)$ who used the terms of individualism and collectivism to describe possible forms of relationships between individuals and the groups to which they belong. According to him, individualism pertains to a society in which the ties between individuals are loose and everyone is expected to look only after himself or herself and his or her immediate family. This definition is obviously narrower than those used in political and philosophical literature.

By identifying related theories and constructs, Waterman (1984) tried to put the definition of individualism into a larger psychological context. According to his ideas, normative or ethical approaches to individualism share four features: (i) being true to one's self (as in Maslow's theory of self-actualization); (ii) freedom of choice within the constraints of the like freedom of others; (iii) personal responsibility which accompanies a sense of being a causally effective agent; and (iv) universality, which involves respect for the integrity of others (cf. Madden, 2001).

Different researchers have suggested more diverse attributes of individualism. Hsu (1983), for instance, considered self-reliance, competitiveness, aggressive creativity, conformity, insecurity, large military expenditures, prejudice toward different racial and religious groups, and unrealistic interpersonal and international relations as the defining features of individualism. According to Ho and Chiu (1994), value of the individual, autonomy, individual responsibility, individual achievement, and self-reliance were the important components of individualism. However, it is not clear whether all these attributes are obligatory components of individualism or simply related concepts.

Aiming to provide a common ground for the research on individualism and collectivism in psychology, Hui and Triandis (1986) surveyed a sample of cross-cultural researchers from all parts of the world on their perception of the meaning of individualism and collectivism. The results of the survey converged, suggesting that there is consensus about the meaning of the dimension. Accordingly, Hui and Triandis (1986) argued that individualism can be defined as (i) the subordination of the goals of the collectivities to individual goals, (ii) a sense of independence, and (iii) lack of concern for others.

In later research, Triandis and his colleagues refined and modified this definition by listing four basic attributes of individualism and/or individualists. Namely, (i) individualists are loosely linked individuals who view themselves as independent of collectives; their self is defined almost entirely in individual terms; (ii) individualists are primarily motivated by their own preferences, needs, rights, and the contracts they have established with others; (iii) individualistic individuals emphasize rational analyses of the advantages and disadvantages to associating with others; and (iv) their personal goals are more important than in-group goals. Individualistic persons are also emotionally detached from their in-groups, and in-groups are perceived as more heterogeneous than out-groups. Social control depends more on guilt than on shame and reflects contractual arrangements. Individualists are ready to confront in-group members with whom one disagrees; competition, and personal fate are emphasized; internal control is high; values are personal (e.g. creative, brave, happy); and there is a congruence between private self and public self (Triandis, 1990, 1995; Triandis et al., 1990, 1993).

A further theoretical and operational elaboration of the constructs was suggested by Singelis and his colleagues (1995). They proposed that the most important attributes of 
individualism (and collectivism) are the horizontal and vertical aspects of social relations (see also Triandis, 1993, 1995). Following the idea that there are four kinds of self, independent and interdependent (Markus \& Kitayama, 1991) and same and different, Singelis and colleagues (1995) proposed a new typology of the combinations of these four types of self-namely, vertical and horizontal individualism and vertical and horizontal collectivism. More specifically, in horizontal individualism, people want to be unique and distinct from groups but they are not especially interested in becoming distinguished or having a higher status. In vertical individualism, people are competitive-they want to become distinguished and have a higher status than the others (Triandis \& Gelfand, 1998).

In his research on cultural values, Schwartz $(1990,1992,1994)$ has also tried to refine the meaning of individualism-collectivism from another angle, both at the individual and cultural levels. In his critique of the individualism-collectivism dichotomy, Schwartz (1990) argued that the values of self-direction, stimulation, and universalism appear to be more important to the average person in individualist societies. In his later article he noted that at the individual-level, the value types of power, achievement, hedonism, stimulation, and self-direction reflect individual interests.

\section{Target/context-specific nature of individualism and collectivism}

The idea that individualism and collectivism are target-specific constructs ${ }^{2}$ was first introduced by Hui and Triandis (1986), who argued that at the individual level many different kinds of individualism and collectivism could be identified according to the targets of interpersonal concern (e.g. spouse, parents, kin, family, neighbours, friends, coworkers/classmates, unknown persons/acquaintances). According to their view, an individual might be concerned and emotionally involved with one particular group (e.g. kin) but not with others (e.g. friends or co-workers). However, the measurement of these target-specific aspects of individualism and collectivism (Hui, 1988; Hui \& Yee, 1994) was only partly successful — confirmatory factor analysis failed to confirm the initial six-factor model corresponding to the proposed relevant target-groups and the internal-consistency reliability coefficients of the revised subscales were marginally satisfactory (cf. Allik \& Realo, 1996).

The Individualism-Collectivism Assessment Inventory (ICAI) of Matsumoto and his associates (Matsumoto, Kudoh, \& Takeuchi, 1996; Matsumoto, Weissman, Preston, Brown, \& Kupperbusch, 1997) examined individualism and collectivism within the four reference groups, family, friends, colleagues, and strangers. Their study showed that common notions of individualism and collectivism between various cultures and ethnicities were indeed carried by specific social relationships.

Using several scales by Triandis and his collaborators for measuring target-specific aspects of individualism and collectivism, Rhee, Uleman, and Lee (1996) made a further attempt to examine whether individualistic and collectivistic attitudes generalize across various in-groups. The results suggested that individualism and collectivism were best represented by a four-factor model with four latent variables: kin collectivism, kin individualism, non-kin collectivism, and non-kin individualism. While collectivism showed only modest generality across kin and non-kin relations and did not differ by cultural group of the respondents, individualism showed generality among Koreans and Asian American but not among European Americans (Rhee, Uleman, \& Lee, 1996).

\footnotetext{
${ }^{2}$ In this pioneer work as well as in a following influential study by Hui (1988), the individualism and collectivism were treated as the opposite poles of a single dimension.
} 
The existence of particular types of individualism or collectivism, however, does not exclude the existence of a general notion or universal core of individualism or collectivism that is shared by all types of individualism or collectivism, respectively. In order to describe the relationships between a general core and specific subforms of collectivism, a hierarchical model of the construct was proposed by Realo, Allik, and Vadi (1997). With respect solely to collectivism, they suggested that collectivism could be best 'understood as a hierarchical concept: the general notion of collectivism is a superordinate concept with many specific subordinate components' (p. 95). They argued that the relationship can be classified into three broad classes or concentric circles according to the social distance: (i) the closest relations between members of family and significant others; (ii) the intermediate relations between neighbours, schoolmates, and co-workers (peers); (iii) the distant relations between a person and the larger social groups and institutions. In the study of collectivistic attitudes among the Estonian population, Realo and colleagues (1997) showed that at least three hierarchically related types of collectivism focusing on relations with family, peers, and society can be distinguished. A later study showed that the three hierarchically related types of collectivism emerged also on a cross-cultural sample including Estonian, NorthAmerican, and two Russian populations (Realo \& Allik, 1999). The hierarchical model of collectivism subsuming several reference group-specific collectivisms was further supported in a recent study by Freeman and Bordia (2001). They claimed, however, that within each in-group domain, individualism and collectivism are polar opposites on a single dimension.

\section{Purpose of the study}

The term 'individualism' originates from a Latin word individuus which means 'indivisible'. Most of the definitions that can be found in the literature rely on the semantical core of individualism - the existence of a single and distinct entity distinguished from a class or group. In comparison with this semantic core, many other properties attributed to individualism seem less fundamental, that is, rather optional than obligatory. For instance, it is widely believed that individualism results in ruthless competition. Yet, although competitiveness appears to be higher in societies in which the rights and goals of individuals are favoured over those of the state, it is certainly not an inevitable result of the individualistic way of living (cf. Waterman, 1981, 1984). Collectivistic societies can be competitive as well.

Analysing previous psychological literature on individualism, we found three different ways in which a person can be 'indivisible' and not abridged to something external to him or her.

(1) Autonomy refers to a person's capacity for independent thinking, judgment, and survival. According to this aspect, a person defines him- or herself as an autonomous and largely independent agent without references to other people, groups, or institutions. It also means that priority is given to one's own aims, decisions, and choices. The theme of autonomy (and independence) has played a central role in most of the discussions about individualism, most notably in the work of Triandis (e.g. Triandis, 1993, 1995) and Schwartz (1994).

(2) Mature self-responsibility. The second theme emphasizes personal responsibility which accompanies a sense of being a causally effective agent. Mature selfresponsibility means that a person accepts responsibility for self and for one's actions 
and has, as a result of this acceptance, confidence in one's abilities. The idea of selfresponsibility as a central attribute of individualism can be found from works of Ho and Chiu (1994) and Waterman (1984), amongst the others.

(3) Uniqueness. This aspect lays emphasis on a person's awareness of being unique, 'only one of its kind'. According to this feature, an individual sees oneself as not being like other people, as being different from the others. The idea of uniqueness being an important component of individualism was most notably introduced by Singelis and colleagues (1995) in their typology of horizontal and vertical types of individualism and collectivism.

In this paper, we attempt to demonstrate the viability of such distinction. In the first part of the article we examine whether the structure exists in one cultural context, Estonia. In this part of the study we also describe the development of a new instrument designed to measure the three components of individualism. To further test the applicability of the three core elements described above, we examine whether the components would relate in hypothesized ways to existing measures of individualism and collectivism and related constructs. Secondly, in line with several other researchers (e.g. Matsumoto, Weissman, Preston, Brown, \& Kupperbusch, 1997; Rhee, Uleman, \& Lee, 1996), we believe that there is a need for scales that measure individualism and collectivism as two separate dimensions and that do so with respect to specific contexts or types of social relationship. Therefore, in the second part of the article we examine whether individualism generalizes across various domains of social relations. More specifically, we study whether we can identify different kinds of individualism according to the three types of social relation with family and close others, peers and friends, state and nation, respectively.

\section{METHOD}

\section{Participants}

The sample for this study consisted of 304 subjects (77 males and 227 females). One hundred and forty-six subjects were the applicants for admission to the Faculty of Social Sciences of the University of Tartu. Seventy-four participants were students of the Department of Psychology of the University of Tartu. The rest of the participants were students from two high schools in the town and county of Tartu as well as adults with diverse socio-demographical background. Subjects' age ranged from 15 to 73 with the mean age 20.4 years $(S D=6.1)$.

\section{Procedure}

Subjects for this study were recruited on a voluntary basis. In most cases, the subjects completed a battery of psychological questionnaires individually; there was no time limit for completing the tests. Data were collected from June to November 2000.

\section{The development of the Three-Component Individualism Scale}

Eighty-nine items for the new measure were generated on the basis of the three core elements of individualism - autonomy (e.g. 'I am disturbed if anyone tries to intervene in my life', 'I don't let others change me'), uniqueness (e.g. 'I am not like other people', 'I have always wanted to somehow differ from others'), and mature self-responsibility (e.g. 'I like situations where I have to decide for myself'). In addition, 71 items were adapted 
from various existing instruments designed to measure individualistic attitudes, values, and behaviours (e.g. the INDCOL Scale, Hui, 1988; Value Profile, Bales \& Couch, 1969; Cultural Value Orientation, Gaines et al., 1997; the Self-Construal Scale, Singelis, 1994; Personal Value Scales, Scott, 1965). Four experts (two graduate students and two faculty members of the Department of Psychology of the University of Tartu) rated each item considering its relevance to the theoretical concept of individualism introduced above. Ratings were given on a three-point scale running from 2 ('the item is very good, it should certainly stay in the item-pool') to 0 ('the item should certainly be excluded from the itempool'). The inter-rater reliability coefficient was 0.72 with an average inter-rater correlation $r=0.40$. The mean score of the experts' ratings was $4.6(\mathrm{SD}=2.2)$ points, ranging from 1 to 8 points. On the basis of the experts' ratings - the baseline criterion for including the item in the final item-pool was 4 points - 105 items were selected for the final item-pool. The 105 items were carefully checked one more time and, as a result, 13 clearly synonymous statements were eliminated from the list. Thus, the final pool of items comprised of 81 items designed for this project and 11 items from the existing scales. The participants of this research were asked to indicate their agreement with the items on a sixpoint Likert-type agreement-disagreement scale.

Among the 92 items, 11 statements were oppositely worded so that the agreement with these statements indicated low individualism. It is important to emphasize here that the development of the Three-Component Individualism Scale followed a theoretical assumption of individualism and collectivism being two separate unipolar factors at the individual level. Accordingly, the oppositely worded items such as 'I don't like situations where I have to decide for myself' or 'I don't differ from thousands of other people', for instance, were included in the item-pool not as indicators of collectivism but as indicators of low individualism. In other words, the oppositely worded items were not conceptually but linguistically reversed, measuring one's lack of individualistic tendencies.

\section{Other measures}

In order to relate the new individualism measure to the other measures of individualism and collectivism and related constructs, three instruments were used in this research. In principle, we tried to select scales with high reliability and validity that have been used in studies in recent years. Yet, our final choice of measures depended first and foremost on availability in Estonian. Therefore, the ESTCOL Scale, the Horizontal and Vertical IndividualismCollectivism Scale, and the Portrait Values Questionnaire were used to examine relationships between various measures of individualism and collectivism.

\section{The ESTCOL scale}

This scale (Realo, Allik, \& Vadi, 1997) measures three interrelated, yet distinguishable subtypes of collectivism focused on relations with family, peers, and society. These types share a common core which is superordinate to these particular forms of collectivism. The scale consisted of 24 items and subjects were asked to indicate their agreementdisagreement with items on a five-point Likert-type scale. The Cronbach alphas of the family-, peers-, and society-related collectivism subscales were $0.83,0.68$, and 0.80 , respectively.

\section{The Horizontal and Vertical Individualism-Collectivism Scale (HVCI)}

The scale was developed by Singelis and colleagues (1995) and adapted to Estonian by Külvet (unpublished Bachelor's Thesis). The Estonian version of the scale consisted of 29 
items; the items were answered on a five-point Likert-type agreement-disagreement scale. The Cronbach alphas were $0.54,0.58,0.67$, and 0.78 for the horizontal collectivism (HC), vertical collectivism (VC), horizontal individualism (HI), and vertical individualism (VI) subscales, respectively.

\section{The Portrait Values Questionnaire (PVQ)}

Values were assessed using the Portrait Values Questionnaire (Schwartz, Melech, Lehmann, Burgess, \& Harris, 2001), a 29-item questionnaire which can be used to assess values at both the individual and cultural level. The scale was adapted to Estonian by Goodwin et al. (2002). Respondents were presented with 29 descriptions of imaginary individuals and asked 'How much is the person in the description like you?'. The answers were given on a six-point scale ranging from not like me at all to very much like me. The scale items were divided into ten value types (measuring hedonism, self-direction, stimulation, conformity, tradition, security, universalism, benevolence, achievement, and power) then formed into two second-order value dimensions, (i) openness to change versus conservation and (ii) self-enhancement versus self-transcendence. The Cronbach alphas of the value types ranged from 0.51 (self-direction and tradition) to 0.77 (achievement). Internal consistency coefficients of the second-order value types were $0.75,0.76,0.79$, and 0.74 for openness to change, conservation, self-enhancement, and self-transcendence.

\section{The development of the Context-Specific Individualism Scale}

In order to examine whether different kinds of individualism can be identified in relation to the three types of social relationship, 12 central attributes of individualism were selected from the item-pool of 92 items that was compiled for developing the Three-Component Individualism Scale. Following the theoretical conceptualization of individualism as introduced above, the 12 attributes were chosen to tap the three facets of individualism: autonomy, uniqueness, and mature self-responsibility. More specifically, the items were the following: autonomy — 'to be independent in your decisions and actions', 'to do things your own way', 'not to depend on them', 'to put first your personal goals and interests', 'not to intervene in their lives and not to let them intervene in your life'; selfresponsibility — 'to take responsibility for your actions/behaviour'; 'to maintain your own self', 'to guide/direct your own life', 'to admit your mistakes'; and uniqueness - two reversed items: 'to be similar to them/to be like they are', 'to identify yourself with them', and 'to stick out from them'. ${ }^{3}$ The participants were asked to rate how important each given principle or value was for them in their relations with (i) family and close others, (ii) friends and peers, and (iii) state and nation, using a seven-point scale running from absolutely unimportant to very important.

\section{RESULTS}

\section{The Three-Component Individualism Scale}

A principal component analysis of the 92 items followed by a varimax rotation revealed that although 25 factors had eigenvalues above one, only the first four factors contained at least eight to ten items with substantial and unique loadings. When the three- and

\footnotetext{
${ }^{3}$ From the methodological point of view, the new scale bears some resemblance to the IndividualismCollectivism Interpersonal Assessment Inventory developed by Matsumoto and his colleagues (1997).
} 
four-factor solutions were compared, the three-factor solution clearly provided the best possible simple structure: 65 items had a primary, substantial loading on one factor only, 15 items had secondary loadings, and 12 items did not significantly (below -0.30 or above 0.30 ) load on any factor. The three factors focused around the three main themes of individualism - autonomy, mature self-responsibility, and uniqueness. In the case of the four-factor structure, only 52 items had a primary loading on one factor, 28 items had secondary loadings, and 12 items had no significant loadings on any factor. As in the threefactor structure, the first three factors concentrated on the themes of autonomy, mature self-responsibility, and uniqueness. The fourth factor combined items that mostly reflected strivings for autonomy and uniqueness as well as tendencies to focus on one's inner thoughts and feelings. Thus, the three-factor solution appeared to provide a better representation of the data on psychometric and contentual grounds.

In order to develop subscales to measure the three components of individualism, the most salient representatives (an item had a significant loading on this particular factor but a near-zero loading on all other factors) for each of these three factors were selected from the list of 92 items. Table 1 shows the items and factor loadings of the varimax-rotated three-factor solution for a set of 24 items. The obtained factor structure, accounting for $40.7 \%$ of the total variance, was rather simple, with each item loading above 0.30 on one factor only. The three-factor structure was also very stable, remaining invariable under different factor extraction (e.g. principal factor analysis with centroid and principal axis method) and rotation (e.g. biquartimax, quartimax, equamax) techniques. To check the correspondence between the three-factor structures of the 24 and of the original 92 items, the correlations between factor scores of the two solutions were calculated across all participants of this research. The correlations for the first two factors were 0.87 and 0.82 (both significant at $p<0.001$ level), showing a fairly good equivalence between the original and final three-factor structures. The correlation for the third factor was somewhat lower, $r=0.58(p<0.001)$; this may be due to the fact that in the factor-structure of the 92 items, the third factor included not only items measuring uniqueness but also items that referred to focusing on one's inner thoughts and feelings. The items which clearly reflected private self-consciousness (e.g. 'I often think about myself', 'I am always trying to figure myself out' etc) were not chosen for the final set of 24 items.

On the basis of the three-factor solution of the 24 items, we developed three subscales for measuring autonomy (ten items), mature self-responsibility (seven items), and uniqueness (seven items). The Cronbach alphas for the Autonomy, Self-Responsibility, and Uniqueness subscales were $0.79,0.76$, and 0.74 , respectively. The intercorrelations between the three subscales (defined as sum scores of the items divided by the number of items in each subscale) were moderate: between Autonomy and Self-Responsibility, $r=0.20$; for Autonomy and Uniqueness, $r=0.30$; and for Self-Responsibility and Uniqueness, $r=0.23$ (all correlations significant at $p<0.05$ ).

Only the Uniqueness subscale was weakly and negatively correlated with age, $r=-0.17, p<0.05$. In general, both men and women received the highest scores on the Autonomy subscale and the lowest scores on the Self-Responsibility subscale. Yet, the General Individualism Index (Autonomy + Self-Responsibility + Uniqueness) was slightly higher for men $(m=13.7, \mathrm{SD}=1.5)$ than for women $(m=13.1, \mathrm{SD}=1.5)$, $t(296)=2.4, p=0.02$; men $(m=4.6, \mathrm{SD}=0.8)$ also scored somewhat higher than women $(m=4.4, \mathrm{SD}=0.8), t(298)=2.1, p=0.04$ on the Uniqueness subscale. Such results conform to several previous findings (e.g. Hui, 1988; Singelis, Triandis, Bhawuk, \& Gelfand, 1995; Triandis, Chan, Bhawuk, Iwao, \& Sinha, 1995), according to which there 
Table 1. Factor loadings of the Three-Component Individualism Scale

\begin{tabular}{|c|c|c|c|c|}
\hline \multirow[t]{2}{*}{ Subscale and item } & \multicolumn{3}{|c|}{ Factor loadings } & \multirow[b]{2}{*}{$R^{2}$} \\
\hline & Factor 1 & Factor 2 & Factor 3 & \\
\hline \multicolumn{5}{|l|}{ Autonomy } \\
\hline I am disturbed if anyone tries to intervene in my life & 0.53 & -0.06 & 0.10 & 0.27 \\
\hline When making decisions, I primarily follow my own needs & 0.55 & 0.22 & 0.12 & 0.31 \\
\hline My interests and goals are most important to me & 0.48 & -0.11 & 0.19 & 0.29 \\
\hline I want to decide myself about things related to my life & 0.61 & 0.18 & 0.10 & 0.44 \\
\hline I usually do as I think is right to do & 0.57 & 0.07 & 0.05 & 0.32 \\
\hline All in all, I don’t represent anyone except myself & 0.57 & -0.01 & -0.05 & 0.30 \\
\hline My life is determined by my own decisions and choices & 0.59 & 0.11 & 0.06 & 0.36 \\
\hline I don't let others change me & 0.66 & 0.15 & 0.00 & 0.39 \\
\hline I place personal freedom above all other values ${ }^{\mathrm{a}}$ & $\mathbf{0 . 5 3}$ & 0.10 & 0.17 & 0.32 \\
\hline $\begin{array}{l}\text { I usually do what I feel is best for me, no matter what } \\
\text { others say }{ }^{\text {b }}\end{array}$ & 0.70 & 0.01 & 0.07 & 0.43 \\
\hline \multicolumn{5}{|l|}{ Self-Responsibility } \\
\hline At meetings, I always speak out about important issues & 0.11 & 0.65 & 0.09 & 0.39 \\
\hline $\begin{array}{l}\text { Most of the important decisions in my life have been } \\
\text { determined by fate and not by my own will }\end{array}$ & -0.05 & 0.51 & 0.00 & 0.24 \\
\hline I am an enterprising and capable person & 0.15 & 0.63 & 0.21 & 0.36 \\
\hline $\begin{array}{l}\text { If I have an opinion about something, I am not afraid } \\
\text { of saying it out }\end{array}$ & 0.20 & 0.58 & 0.04 & 0.35 \\
\hline $\begin{array}{l}\text { I don't like situations where I have to be responsible for } \\
\text { something important }{ }^{\mathrm{R}}\end{array}$ & -0.21 & 0.71 & -0.01 & 0.45 \\
\hline $\begin{array}{l}\text { I am considered to be a person who has an independent } \\
\text { opinion }\end{array}$ & 0.13 & 0.66 & 0.20 & 0.41 \\
\hline $\begin{array}{l}\text { I don't like situations where I have to decide for myself }{ }^{R} \\
\text { Uniqueness }\end{array}$ & 0.14 & 0.65 & -0.05 & 0.44 \\
\hline I have always wanted to somehow differ from others & 0.15 & -0.02 & 0.72 & 0.41 \\
\hline I have qualities other people are not aware of & 0.27 & -0.06 & 0.39 & 0.21 \\
\hline I like being distinguished from the crowd & 0.07 & 0.19 & 0.74 & 0.49 \\
\hline I am not like other people & 0.22 & 0.06 & 0.60 & 0.35 \\
\hline I don't differ from thousands of other people ${ }^{R}$ & -0.16 & 0.08 & 0.58 & 0.31 \\
\hline It is important to me to stand out from others & -0.01 & 0.28 & 0.58 & 0.37 \\
\hline By my character, I think I am a distinct person & 0.24 & -0.03 & 0.67 & 0.37 \\
\hline
\end{tabular}

Note. $N=298$. Loadings greater than 0.30 are boldface. $R^{2}=$ variable communalities. The items are approximate translations from Estonian to English. ${ }^{\mathrm{R}}$ Item reversed for scoring.

${ }^{a}$ Item adapted from the Cultural Value Orientation of Gaines et al. (1997).

bItem adapted from Marshall's (1997) individualism scale.

appears to be a slight difference between men and women (with women frequently scoring lower than men on certain aspects of individualism) that is often too small to be of any substantial or practical importance.

\section{Correlations between the Three-Component Individualism Scale and other measures}

To further establish the applicability of the three core components of individualism, it is important to demonstrate that the components relate, in hypothesized ways, to existing measures of individualism and collectivism and other related constructs. For that purpose, the relationships between the subscales of the Three-Component Individualism Scale and various measures of individualism and collectivism and values were examined. Given the 
theoretical arguments presented in the 'Introduction' section, we expected that all components of individualism would be positively related to horizontal (HI) and vertical individualism (VI), whereas the relationship would be extra strong between Uniqueness and HI. Following the assumption that individualism and collectivism are not the opposite poles of the same dimension, we also hypothesized that the three core elements would be unrelated to horizontal (HC) and vertical collectivism (VC) as well as to family-, peer-, and society-related collectivism. Finally, the components of individualism were expected to be positively related to the value types of self-direction, stimulation, achievement, and power as well as to the respective second-order value dimensions openness to change and self-enhancement that, according to Schwartz (1992), all serve individual interests.

The pattern of correlations between the subscales of the Three-Component Individualism Scale and other measures is presented in Table 2. As can be seen from this table, the three developed scales are related to both vertical and horizontal aspects of individualism. The General Individualism Index, however, appeared to be more strongly related to HI ( $r=0.61$; lower and upper bounds for 95\% confidence interval $=0.54$ and 0.68 , respectively) than to VI $(r=0.32$; lower and upper bounds for $95 \%$ confidence

Table 2. Correlations between the subscales of the Three-Component Individualism Scale and the HVCI Scale, the ESTCOL Scale, and the Portrait Values Questionnaire

\begin{tabular}{|c|c|c|c|c|}
\hline \multirow[t]{2}{*}{ Scale } & \multicolumn{4}{|c|}{ The Three-Component Individualism Scale } \\
\hline & Autonomy & $\begin{array}{l}\text { Self- } \\
\text { responsibility }\end{array}$ & Uniqueness & $\begin{array}{c}\text { General } \\
\text { Individualism } \\
\text { Index }\end{array}$ \\
\hline \multicolumn{5}{|l|}{ The HVCI } \\
\hline Horizontal individualism & $0.37 * * *$ & $0.34 * * *$ & $0.56 * * *$ & $0.61 * * *$ \\
\hline Vertical individualism & $0.23 * * *$ & $0.19 * * *$ & $0.26 * * *$ & $0.32 * * *$ \\
\hline Horizontal collectivism & -0.05 & -0.04 & -0.01 & -0.05 \\
\hline Vertical collectivism & -0.06 & -0.03 & -0.10 & -0.10 \\
\hline \multicolumn{5}{|l|}{ The ESTCOL Scale } \\
\hline Family & 0.07 & -0.11 & $-0.13 *$ & -0.09 \\
\hline Peers & -0.04 & $-0.16^{* *}$ & -0.10 & $-0.16 * *$ \\
\hline Society & -0.07 & -0.00 & -0.01 & -0.03 \\
\hline General Collectivism Index & -0.01 & -0.11 & -0.10 & $-0.12 *$ \\
\hline \multicolumn{5}{|l|}{ The PVQ } \\
\hline Openness to change & $0.32 * * *$ & $0.32 * * *$ & $0.42 * * *$ & $0.51 * * *$ \\
\hline Stimulation & $0.21 * * *$ & $0.18 * *$ & $0.33 * * *$ & $0.34 * * *$ \\
\hline Self-direction & $0.34 * * *$ & $0.38 * * *$ & $0.34 * * *$ & $0.51 * * *$ \\
\hline Self-enhancement & $0.20 * * *$ & $0.19 * * *$ & $0.41 * * *$ & $0.38 * * *$ \\
\hline Power & $0.15 * *$ & 0.10 & $0.26 * * *$ & $0.25 * * *$ \\
\hline Achievement & $0.14 *$ & $0.30 * * *$ & $0.45 * * *$ & $0.44 * * *$ \\
\hline Hedonism & $0.18 * *$ & 0.05 & $0.25 * * *$ & $0.22 * * *$ \\
\hline Conservation & 0.08 & -0.04 & $-0.14 *$ & -0.06 \\
\hline Conformity & 0.02 & -0.07 & $-0.15 * *$ & -0.11 \\
\hline Tradition & 0.06 & $-0.16^{* *}$ & $-0.21 * * *$ & $-0.17 * *$ \\
\hline Security & 0.11 & 0.11 & 0.01 & 0.11 \\
\hline Self-transcendence & $0.18 * *$ & 0.10 & 0.03 & $0.14 *$ \\
\hline Universalism & $0.12 *$ & 0.01 & 0.00 & 0.05 \\
\hline Benevolence & $0.19 * * *$ & $0.18 * *$ & 0.07 & $0.20 * * *$ \\
\hline
\end{tabular}

Note. The HVCI = The Horizontal and Vertical Individualism-Collectivism Scale; The PVQ=The Portrait Values Questionnaire.

$* * * p<0.001 ; * * p<0.01 ; * p<0.05$. 
interval $=0.22$ and 0.42 , respectively). Among the three components of individualism, Uniqueness showed the highest correlation with HI, as expected.

All correlations between the components of individualism and horizontal-vertical aspects of collectivism were near zero. Uniqueness showed a meagre negative correlation with family-related and Self-Responsibility with peer-related collectivism, respectively; the General Individualism Index was weakly negatively correlated both with peer-related collectivism and with the General Collectivism Index (family+peers+society). Thus, our results supported the view of individualism and collectivism being rather two separate dimensions than one bipolar factor.

The three components of individualism exhibited a relatively similar pattern of positive correlations with the high-order value dimension openness to change as well as to its subtypes stimulation and self-direction. ${ }^{4}$ Yet, while Uniqueness was quite strongly and Autonomy moderately correlated with the value dimension self-enhancement and its subordinate value types such as power, achievement, and hedonism, Self-Responsibility was positively correlated only with achievement and not with power and hedonism. Furthermore, a differentiated pattern of correlations emerged between the three components of individualism and the value types that, according to Schwartz (1992), serve collective interests. For instance, Uniqueness had a negative relationship with the value types of conformity and tradition (conservation); Self-Responsibility was negatively related to tradition and positively to benevolence; whereas Autonomy was positively correlated only with universalism and benevolence (self-transcendence). These patterns of correlations suggest that the three components of individualism are related because they all emphasize self-direction, stimulation, and achievement but are distinct in terms of their emphasis on power, hedonism, universalism, and benevolence, for instance.

\section{Context-specific versus general nature of individualism}

In this part of the study we examined whether individualism generalizes across specific contexts or types of social relationship, namely, across relations with family and close others; friends and peers; state and nation. More specifically, we investigated (i) whether the inclusion of specific types of social relationship alters the number and/or interpretation of the resulting facets of individualism and (ii) whether people differ in their individualistic beliefs with relation to various social contexts.

To this aim, we studied the data that were obtained using the Context-Specific Individualism Scale. Since this data-set has three modes (i.e. contexts by individualism attributes by persons), a three-mode principal component analysis (3MPCA; Kroonenberg \& De Leeuw, 1980) was applied, which is a technique specifically designed for the analysis of three-mode data. As a generalization of the standard (two-mode) principal component analysis, 3MPCA summarizes the information in each of the three modes through a relatively small number of components and describes the relations between these components (see Kiers \& Van Mechelen, 2001; Van Mechelen \& Kiers, 1999).

Technically speaking, 3MPCA summarizes the contexts by a number (\#) of unobserved context components $(C C)$, the individualism attributes by a number of unobserved attribute components $(A C)$ and the persons by a number of unobserved person components $(P C)$. The context, attribute, and person components are further linked in a so-called core

\footnotetext{
${ }^{4}$ While interpreting the correlations between the subscales of the Three-Component Individualism Scale and the Schwartz value types it should be kept in mind that the magnitude of several correlations is limited by low internal consistencies of the value types (especially of traditionalism, stimulation, power, self-direction, and conformity).
} 
array of order $\# C C \times \# A C \times \# P C$. For example, the core entry that represents the relations among $C C 1, A C 1$, and $P C 1$ indicates to what extent a person scoring high on $P C 1$ will value an individualism attribute scoring high on $A C 1$ in a context scoring high on $C C 1$.

In this study, we fitted 3MPCA models with \#CC ranging from one to three, \#AC ranging from one to five and $\# P C$ ranging from one to five to our data (an extensive discussion of how to apply 3MPCA on a dataset may be found in the work of Kiers \& Van Mechelen, 2001). ${ }^{5}$ A generalized scree test (Timmerman \& Kiers, 2000) suggested we consider two solutions, Model 1 with $\# C C=1$, \#AC=4, \#PC=4 and Model 2 with $\# C C=2, \# A C=3$, \#PC $=4$, both models accounting for about $38.6 \%$ of the total variance. For both solutions, split-half stability and interpretability checks were performed. The two solutions appeared to be equally stable over split halves and were both relatively easily interpretable. Thus, in the following paragraphs, both solutions will be described in order to find answers to the above mentioned research questions.

First, Model 1 is examined, which summarizes data by one context, four attribute, and four person components. With respect to the contexts, all three domains of social relationships have about equal loadings on the sole $C C(0.55,0.60$, and 0.57 for family, friends and peers, state and nation, respectively). This indicates that, according to the model, the three contexts did not elicit different individualistic beliefs from the participants of our study.

Table 3 presents the scores of the 12 individualism items on the four attribute components. $A C 1$ can be interpreted as uniqueness since all three items emphasizing uniqueness have high loadings on this component. ${ }^{6}$ As five attributes measuring autonomy have significant loadings on $A C 2$, it can be called autonomy. Given the high loadings of the two self-responsibility items, $A C 3$ can be labelled self-responsibility. Finally, $A C 4$ can be interpreted as self-maintenance, since the item 'to maintain your own self' has the highest loading on this component.

Figure 1 shows line plots of core values for the four person components. More specifically, it shows the importance of the four attribute components (i.e. the themes of individualism) for persons with a high positive score on the respective component. From these plots it can be read that respondents scoring high on $P C 1, P C 2, P C 3$, and $P C 4$ mainly value uniqueness, autonomy, self-responsibility, and self-maintenance, respectively.

Model 2 differentiates between two context, three attribute, and four person components. With loadings of $0.54,0.61$, and $0.57, C C 1$ can be described as a general context component. Given the loadings of $-0.56,-0.24$, and 0.79 for family, friends and peers, state and nation, respectively, CC2 may be interpreted as distance of social relationships (ranging from close relations with one's family to more remote and distant relations with state and nation). In view of the amount of variance that the two context components account for $(32.8 \%$ and $5.7 \%$, respectively), however, one may conclude that the majority of the variance the model accounts for (38.6\%) can be explained without differentiating between various contexts.

Regarding the attribute components, Table 3 clearly shows that the three components reflect the proposed facets of individualism; therefore, the attribute components are labelled uniqueness, autonomy and self-responsibility, respectively.

\footnotetext{
${ }^{5} \mathrm{~A}$ detailed description of the rationale behind the choices made in the present analysis can be obtained from the authors.

${ }^{6}$ In both models, contrary to our theoretical expectations, the item 'To stick out from them' had high negative loadings on the uniqueness attribute component, meaning that people who value uniqueness do not want to be similar to specific others and do not want to identify themselves with these people, as well as not wanting to stand out from them.
} 


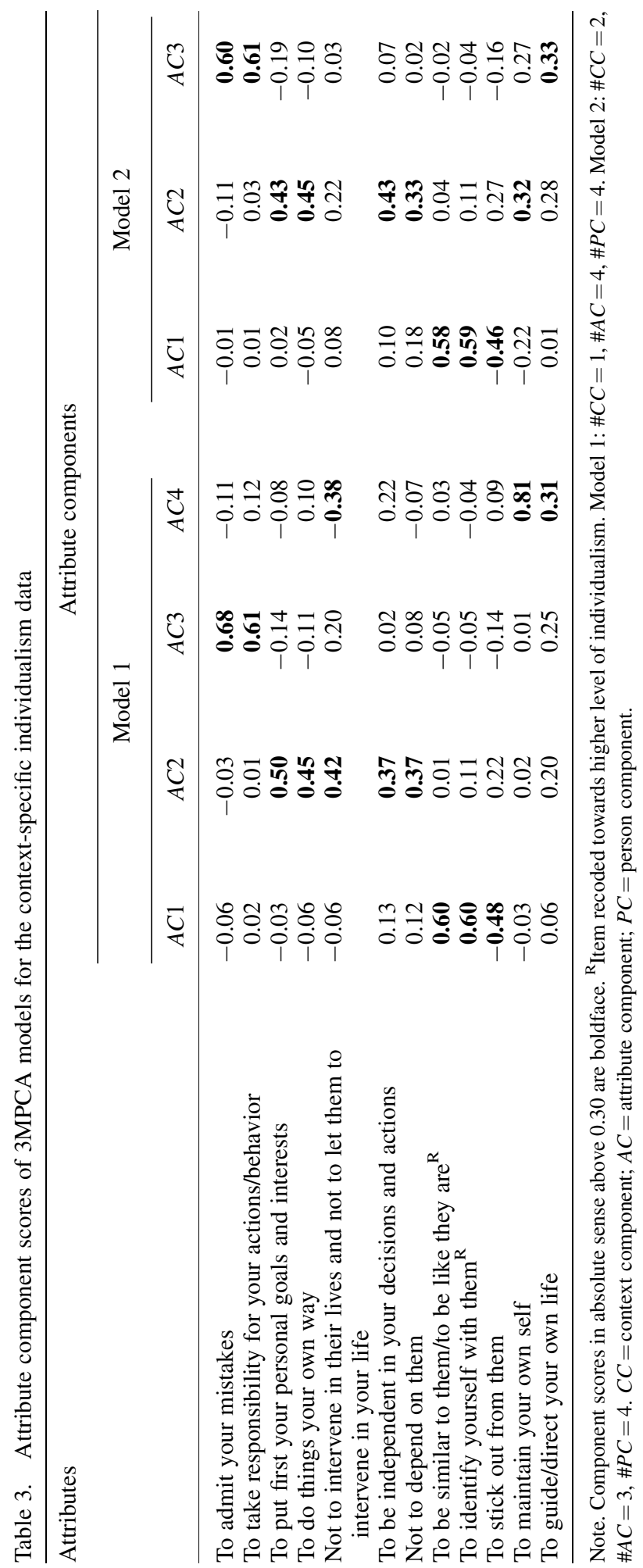



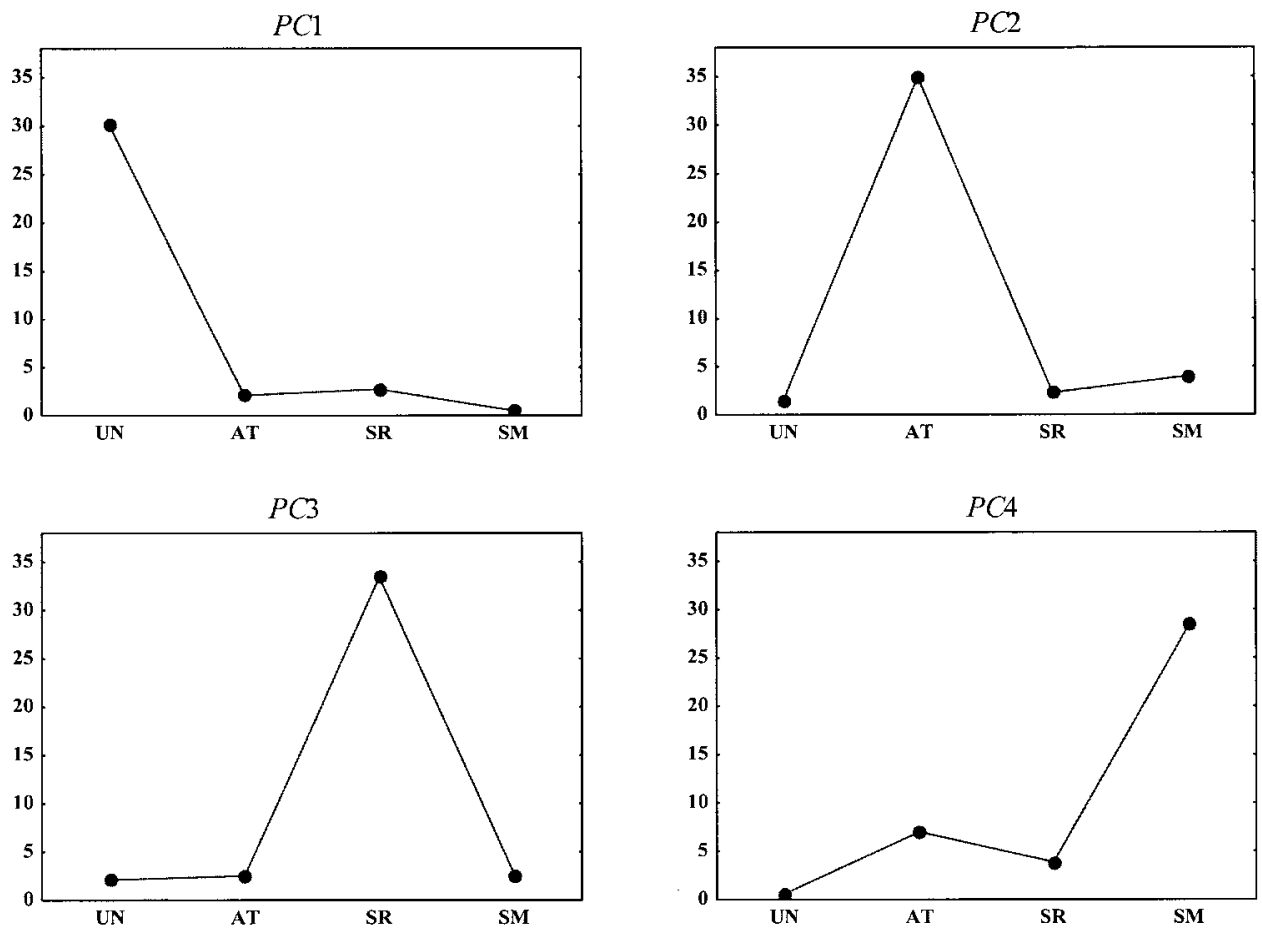

Figure 1. Line plots of core values, resulting from 3MPCA Model $1(\# C C=1, \# A C=4, \# P C=4)$ for the context-specific individualism data. Each line plot gives core values for one person component $(P C)$; the core values pertain to the four attribute components $(\mathrm{UN}=$ uniqueness, $\mathrm{AT}=$ autonomy, $\mathrm{SR}=$ self-responsibility, and $\mathrm{SM}=$ self-maintenance).

Given the profiles in Figure 2, the person components can be interpreted in the following way. Persons who have high positive scores on $P C 1, P C 2$, and $P C 3$ value uniqueness, autonomy, and self-responsibility in all three contexts, respectively. $P C 4$, on the other hand, represents participants who consider the distance of the social relationships important in their ratings of individualism attributes: the more distant the domain of social relationships, the more the participants with high positive scores on this component value their autonomy and, to a somewhat lesser extent, uniqueness. The participants who have high negative scores on this component, however, tend to emphasize their autonomy and uniqueness in less distant social relationships such as with family and close others.

To sum up our findings, we may conclude the following.

(i) The inclusion of three contexts in the measurement of individualism did not alter the individualism facets much: except for the splitting up of the self-responsibility theme into two components (i.e. self-responsibility and self-maintenance) in Model 1, the three core elements of individualism were retrieved in both sets of individualism attribute components.

(ii) The individualistic beliefs of the respondents did not differ much across the three domains of social relations. Only in Model 2, one person component (PC4) was found (accounting for $5.6 \%$ of variance), for which people who scored high on this component also differentiated among the three domains of social relationships in their ratings of individualism attributes. 

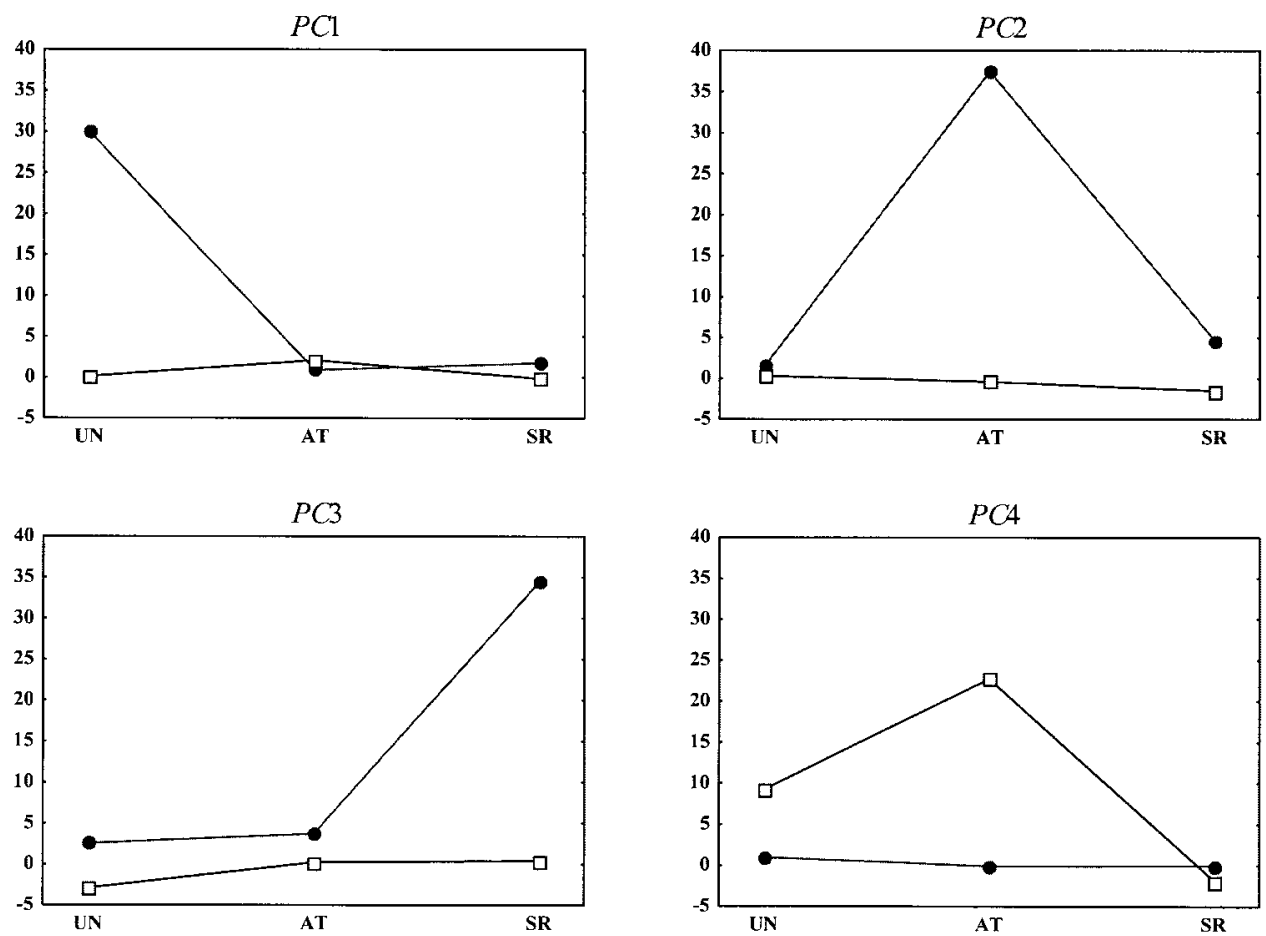

Figure 2. Line plots of core values, resulting from 3MPCA Model $2(\# C C=2, \# A C=3, \# P C=4)$ for the context-specific individualism data. Each line plot gives core values for one personality component $(P C)$; the core values pertain to the three attribute components ( $\mathrm{UN}=$ uniqueness, $\mathrm{AT}=$ autonomy, $\mathrm{SR}=$ self-responsibility) crossed with the two context components (general, indicated by filled circles, and distance of social relationships, indicated by unfilled boxes).

\section{DISCUSSION}

Recent theorizing and empirical research suggests that, at the individual level, both individualism and collectivism are most likely multifaceted constructs-each composed of several interrelated but still distinct subforms. In this article, following an assumption that individualism and collectivism are nearly orthogonal factors, we have further established that the distinction between different components of individualism is important. Although in the past researchers have identified numerous features and component ideas of individualism (see Kagitçibasi, 1997, for a review), we argue that at the intermediate level of description (neither too broad nor too particular) one may talk about three major themes or aspects of individualism that emphasize autonomy, uniqueness, and mature self-responsibility, respectively.

\section{Three components of individualism}

A new scale was developed to measure the three aspects of individualism, which demonstrated both the reasonable internal consistency reliability as well as convergent and divergent validity with several other measures of individualism and collectivism and related constructs. We found that all three components of individualism were positively correlated with vertical and horizontal individualism, and with the second-order value type openness to change and its subtypes emphasizing stimulation and self-direction. These 
relationships suggest that our measure reflects indeed some aspects of the general notion of individualism. At the same time, the three components of individualism were not related to horizontal and vertical collectivism, supporting the conceptualization of the individualism and collectivism constructs as two separate dimensions: individuals who rate themselves as being horizontally or vertically collectivistic may also emphasize their autonomy, uniqueness, and responsibility for their deeds, for instance. Although Uniqueness and SelfResponsibility exhibited negative correlations with the family- and peer-related collectivisms, respectively, the correlations were relatively small and irregular. Therefore, we can argue that knowing a person's score on a scale measuring horizontal or familyrelated collectivism, for instance, tells us little if anything at all about one's individualistic tendencies.

Our data also demonstrated that the three components of individualism were differentially related to several other constructs measured in this study. In contrast to Uniqueness, people high in Autonomy and Self-Responsibility stressed values related to benevolence, that is 'preserving and enhancing the welfare of people to whom one is close' (Smith \& Schwartz, 1997, p. 86). Autonomy was also related to universalism, meaning that autonomous people tended to stress the understanding, tolerance, and protection of the welfare of all people and nature. Such results clearly suggest that potentialities for both individualism and altruism may be present in each person: 'an increasingly mature sense of self is contingent on interpersonal relationships; conversely, the continued development of increasingly mature interpersonal relationships is contingent on mature self-definition' (Guisinger \& Blatt, 1994, p. 104).

\section{Context-specific versus context-free nature of individualism}

Another important question we addressed in this paper is whether individualism and collectivism are situation or target specific or have trait like generality over contexts and targets. Although in several studies (Allik \& Realo, 1996; Freeman \& Bordia, 2001; Hui, 1988; Hui \& Triandis, 1986; Matsumoto, Weissman, Preston, Brown, \& Kupperbusch, 1997; Realo, Allik, \& Vadi, 1997; Rhee, Uleman, \& Lee, 1996) individualistic and collectivistic tendencies have appeared to be target specific, there has been a general tendency to attribute trait like characteristics to individualism and collectivism and to see them as shaped mainly by personality or culture (cf. Kagitçibasi, 1997; Realo, 1998, for a review). Our previous work (Realo, Allik, \& Vadi, 1997) with regard to collectivism showed that various kinds of collectivism can be distinguished from one another by the type of social relation they focus on (e.g. family, peers, and society) and can be united on the basis of the two Big Five factors, Closedness (as opposed to Openness to Experience) and Agreeableness.

In this study, we examined whether individualism in one domain of social relations generalizes toward the other types of social relationship using the data that were obtained by the Context-Specific Individualism Scale. First, we found that the three core elements of individualism were retrieved also when different types of social relationship were included in the measurement. In the first model, however, one of the components of individualism - self-responsibility - was split into two subtypes, responsibility and selfmaintenance. Such finding suggests that the three components of individualism may be divided into more specific, lower-order subthemes.

The results of the 3MPCA also indicated that participants' individualistic tendencies did not differ much while measured with regard to three different types of social relationship. 
Except for one person component (PC4) in Model 2, people tended to be similarly individualistic in their relations with family and close others; friends and peers; state and nation. Yet, the emergence of PC4 in the second model (see Figure 2) shows that there appears to be a certain number of people in our sample who consider the distance of social relationships important in their ratings of individualism attributes. While some of these people value their autonomy and uniqueness in relationships with country and nation, the others emphasize the two aspects of individualism in relations with their family and close others.

\section{CONCLUSION}

The results reported in this paper have several implications for future research. The present study provided empirical evidence that it is possible to distinguish between at least three distinct components of individualism - autonomy, self-responsibility, and uniqueness, respectively. Yet, as our research was limited to only one culture, future research should test to what extent the proposed tripartite division of individualism is extendible to other languages and cultures.

Our model of three components of individualism does not, of course, necessarily contest the validity of other models of individualism, such as Triandis' model of horizontal and vertical dimensions of individualism (Triandis \& Gelfand, 1998), for instance. The distinction between three components of individualism - autonomy, uniqueness, and selfresponsibility - should be rather seen as a further specification of the individualism construct, parallel to the horizontal-vertical aspect. Future studies should investigate how the three components of individualism are associated with other constructs such as competitiveness, egoism or locus of control that are often linked to individualism in crosscultural literature.

Future research should be also directed at exploring the generalizability of our results that, by and large, individualistic tendencies did not differ across three types of social relationship. One explanation might lie in the fact that while collectivism explicitly refers to other people or to social relationships with other people, individualism mainly refers to one's self. In other words, one can be autonomous and take responsibility for one's deeds without specific references to other people while one can have collectivist tendencies only towards certain groups of people (e.g. family, relatives, friends). Yet, the finding that there were people in our sample for whom the distance of social relationships was important in describing their individualistic tendencies seems to speak against such interpretation. Thus, it is plausible that in some groups within a culture or in some other cultures the individualistic attitudes are more dependent on a specific context or a type of social relationship they refer to. Rhee, Uleman, and Lee (1996) found, for instance, that individualism showed generality across various in-groups only among Koreans and Asian Americans but not among European Americans. These findings suggest that future studies are needed with diverse populations both within and across cultures to further analyse the context-specific nature of individualistic tendencies.

Beyond this, our study raised several methodological issues. First, the context-specific measurement of individualism as applied in this research calls for a study of contextspecific measurement of collectivism, using a similar kind of S-R (stimulus-response) inventory. Such an approach has been already promoted by Matsumoto and his colleagues (1997), but in their research individualism and collectivism were treated as opposing poles 
of the same dimension. So far, all other context-specific measurements of individualism and collectivism (Hui, 1988; Freeman \& Bordia, 2001; Realo, Allik, \& Vadi, 1997; Rhee, Uleman, \& Lee, 1996) have been conducted using different sets of items to measure individualistic or collectivistic tendencies toward various targets of social relations. In the ESTCOL Scale of Realo, Allik, and Vadi (1997), for instance, items such as 'In life, family interests are most important' and 'A family should have one joint budget' were used to measure family-related collectivism whereas society-related collectivism was measured by items such as 'If necessary, one should die in the name of his/her nation' and 'The interests of nation outweigh the individual interests of its members', for instance. Further research should establish whether and to what extent the context-specific measurements of individualism and collectivism depend on the design of the questionnaire (the INDCOLtype attitude scale versus the S-R-type inventory).

The S-R-type inventories (using the same items to measure individualism or collectivism with relation to specific contexts or relationships) have several advantages over the traditional INDCOL-type attitude scales in which separate sets of items address each context. As Matsumoto, Kudoh, \& Takeuchi (1996) pointed out, for instance, the averaged scores of the INDCOL subscales (Hui, 1988) are not comparable across relationships because the items producing those scores are different. Cross-context or relationship assessment of individualistic or collectivistic tendencies using the same set of items, however, offers a possible solution to this problem. Our study showed that another advantage of the S-R-type inventories in the measurement of individualistic or collectivistic tendencies is that they allow to collect data that can be effectively analysed using three-way techniques (such as the 3MPCA used in our study, for instance). These techniques are gaining more and more popularity in personality research (cf. Kiers \& Van Mechelen, 2001; Van Mechelen \& Kiers, 1999) as they provide excellent possibilities for describing and explaining individual differences in psychological functioning with explicit references to specific contexts and behaviors.

Finally, following the idea that various kinds of collectivism can be distinguished from one another by the type of social relation and united on the basis of relatively stable personality traits as shown in our previous research (Realo, Allik, \& Vadi, 1997), an important question arises here. Namely, how and to what extent are individualistic tendencies related to the basic personality dispositions? So far, there are only a few studies (with relatively diverse and inconsistent findings) that have addressed this issue (see e.g. Dollinger, Preston, O’Brien, \& DiLalla, 1996; Grimm, Church, Katigbak, \& Reyes, 1999; Luk \& Bond, 1993). If individualism (along with collectivism) is treated as an individualdifferences dimension (cf. Church, 2000; Church \& Lonner, 1998), future research should specify how to describe individualism in terms of some combination of basic personality dimensions.

\section{ACKNOWLEDGEMENTS}

This research is based, in part, on a master's thesis by Kati Koido. Preparation of this article was supported by grants F/00/036 and GOA/00/02 from the Research Council of the University of Leuven. We thank Henk Kiers for providing us with the Tucker3.m program to conduct the three-mode principal component analysis. We are grateful to Iven Van Mechelen and to two anonymous reviewers for their most helpful comments and critical remarks on a former version of the manuscript. We also thank Karmen Linnamägi for her assistance during the earlier stages of this study. 


\section{REFERENCES}

Allik J, Realo A. 1996. The hierarchical nature of individualism-collectivism: comments on Matsumoto et al. (1996). Culture and Psychology 2: 109-117.

Bales R, Couch A. 1969. The value profile: a factor analytic study of value statements. Sociological Inquiry 39: 3-17.

Chen CC, Meindl JR, Hunt RG. 1997. Testing the effects of vertical and horizontal collectivism: a study of reward allocation preferences in China. Journal of Cross-Cultural Psychology 28(1): 4470.

Church AT. 2000. Culture and personality: toward an integrated cultural trait psychology. Journal of Personality 68: 651-703.

Church AT, Lonner WJ. 1998. The cross-cultural perspective in the study of personality: rationale and current research. Journal of Cross-Cultural Psychology 29: 32-62.

Dollinger SJ, Preston LA, O'-Brien SP, DiLalla DL. 1996. Individuality and relatedness of the self: an autophotographic study. Journal of Personality and Social Psychology 71: 1268-1278.

Fijneman YA, Willemsen ME, Poortinga YH. 1996. Individualism-collectivism: an empirical study of a conceptual issue. Journal of Cross-Cultural Psychology 27: 381-402.

Freeman MA, Bordia P. 2001. Assessing alternative models of individualism and collectivism: a confirmatory factor analysis. European Journal of Personality 15: 105-121.

Gaines SO Jr, Marelich WD, Bledsoe KL, Steers WN, Henderson MC, Granrose CS, Barajas L, Hicks D, Lyde M, Takahashi Y, Yum N, Rios DI, Garcia BF, Farris KR, Page MS. 1997. Links between race/ethnicity and cultural values as mediated by racial/ethnic identity and moderated by gender. Journal of Personality and Social Psychology 72: 1460-1476.

Goodwin R, Realo A, Kwiatkowska A, Kozlova A, Nguyen Luu LA, Nizharadze G. 2002. Values and sexual behavior in Central and Eastern Europe. Journal of Health Behaviour.

Grimm SD, Church AT, Katigbak MS, Reyes JA. 1999. Self-described traits, values, and moods associated with individualism and collectivism: testing I-C theory in an individualistic (U.S.) and a collectivistic (Philippine) culture. Journal of Cross-Cultural Psychology 30: 466-500.

Guisinger S, Blatt SJ. 1994. Individuality and relatedness: evolution of a fundamental dialectic. American Psychologist 49: 104-111.

Ho DY-F, Chiu C-Y. 1994. Component ideas of individualism, collectivism, and social organization: an application in the study of Chinese culture. In Individualism and Collectivism: Theory, Method and Applications (pp. 137-156). Kim U, Triandis HC, Kagitçibasi C, Choi S-C, Yoon G (eds). Sage: Thousand Oaks, CA.

Hofstede G. 1980. Culture's Consequences: International Differences in Work-Related Values. Sage: Beverly Hills, CA.

Hofstede G. 1991. Cultures and Organizations: Software of the Mind. McGraw-Hill: London.

Hsu FLK. 1983. Rugged Individualism Reconsidered. University of Tennessee Press: Knoxville, TN.

Hui CH. 1988. Measurement of individualism-collectivism. Journal of Research in Personality 22: $17-36$.

Hui CH, Triandis HC. 1986. Individualism-collectivism: a study of cross-cultural researchers. Journal of Cross-Cultural Psychology 17: 225-248.

Hui $\mathrm{CH}$, Yee C. 1994. The shortened individualism-collectivism scale: its relationship to demographic and work-related variables. Journal of Research in Personality 28: 409-424.

Kagitçibasi C. 1994. A critical appraisal of individualism and collectivism: toward a new formulation. In Individualism and Collectivism: Theory, Method, and Applications (pp. 52-65). Kim U, Triandis HC, Kagitçibasi C, Choi S-C, Yoon G (eds). Sage: Thousand Oaks, CA.

Kagitçibasi C. 1997. Individualism and collectivism. In Handbook of Cross-Cultural Psychology: Vol. 3. Social Behavior and Applications (2nd ed., pp. 1-49). Berry JW, Poortinga YH, Pandey J, Dasen PR, Saraswathi TS, Segall MH, Kagitçibasi C (Series eds), Berry JW, Segall MH, Kagitçibasi C (Vol. eds). Allyn and Bacon: Boston, MA.

Kiers HAL, Van Mechelen I. 2001. Three-way component analysis: principles and illustrative application. Psychological Methods 6: 84-110.

Kroonenberg PM, de Leeuw J. 1980. Principal component analysis of three-mode data by means of alternating least squares algorithms. Psychometrika 45: 69-97.

Luk CL, Bond MH. 1993. Personality variation and values endorsement in Chinese university students. Personality and Individual Differences 14: 429-437. 
Lukes S. 1971. The meanings of individualism. Journal of the History of Ideas 32: 45-66.

Madden R. 2001. Individualism, interdependence, and interpersonal conflict. Spintech. Retrieved March 12, 2001, from http://www.spintechmag.com/0103/rm0301.htm

Markus HR, Kitayama S. 1991. Culture and the self: implications for cognition, emotion, and motivation. Psychological Review 98: 224-253.

Marshall R. 1997. Variances in levels of individualism across two cultures and three social classes. Journal of Cross-Cultural Psychology 28: 490-495.

Matsumoto D, Kudoh T, Takeuchi S. 1996. Changing patterns of individualism and collectivism in the United States and Japan. Culture and Psychology 2: 77-107.

Matsumoto D, Weissman MD, Preston K, Brown BR, Kupperbusch C. 1997. Context-specific measurement of individualism-collectivism on the individual level: the Individualism-Collectivism Interpersonal Assessment Inventory. Journal of Cross-Cultural Psychology 28: 743-767.

Realo A. 1998. Collectivism in an individualist culture: the case of Estonia. Trames 2: 19-39.

Realo A, Allik J. 1999. A cross-cultural study of collectivism: a comparison of American, Estonian, and Russian students. Journal of Social Psychology 139: 133-142.

Realo A, Allik J, Vadi M. 1997. The hierarchical structure of collectivism. Journal of Research in Personality 31: 93-116.

Rhee E, Uleman JS, Lee HK. 1996. Variations in collectivism and individualism by ingroup and culture: confirmatory factor analyses. Journal of Personality and Social Psychology 71: 10371054.

Schwartz SH. 1990. Individualism-collectivism: critique and proposed refinement. Journal of Cross-Cultural Psychology 21: 139-157.

Schwartz SH. 1992. Universals in the content and structure of values: theoretical advances and empirical tests in 20 countries. In Advances in Experimental Social Psychology (Vol. 25, pp. 165). Zanna M (ed.). Academic: New York.

Schwartz SH. 1994. Beyond individualism/collectivism: new cultural dimensions of values. In Individualism and Collectivism: Theory, Method, and Applications (pp. 85-119), Kim U, Triandis HC, Kagitçibasi Ç, Choi SC, Yoon G (eds). Sage: Thousand Oaks, CA.

Schwartz SH, Melech G, Lehmann A, Burgess S, Harris M. 2001. Extending the cross-cultural validity of the theory of basic human values with a different method of measurement. Journal of Cross-Cultural Psychology 32: 519-542.

Scott WA. 1965. Values and organizations: a study of fraternities and sororities. Rand McNally: Chicago, IL.

Singelis TM. 1994. The measurement of independent and interdependent self-construals. Personality and Social Psychology Bulletin 20: 580-591.

Singelis TM, Triandis HC, Bhawuk DPS, Gelfand MJ. 1995. Horizontal and vertical dimensions of individualism and collectivism: a theoretical and measurement refinement. Cross-Cultural Research 29: 240-275.

Smith PB, Schwartz SH. 1997. Values. In Handbook of Cross-Cultural Psychology: Vol. 3. Social behavior and applications (2nd ed., pp. 77-118), Berry JW, Segall MH, Kagitçibasi Ç (Vol. eds). Allyn and Bacon: Boston, MA.

Timmerman ME, Kiers HAL. 2000. Three-mode principal components analysis: choosing the number of components and sensitivity to local optima. British Journal of Mathematical and Statistical Psychology 53: 1-16.

Triandis HC. 1990. Cross-cultural studies of individualism and collectivism. In Nebraska Symposium on Motivation (pp. 41-133), Berman J (ed.). University of Nebraska Press: Lincoln, NE.

Triandis HC. 1993. Collectivism and individualism as cultural syndromes. Cross-Cultural Research 27: $155-180$.

Triandis HC. 1995. Individualism and Collectivism. Westview Press: Boulder, CO.

Triandis HC. 2001. Individualism-collectivism and personality. Journal of Personality 69: 907-924.

Triandis HC, Bontempo R, Betancourt H, Bond M, Leung K, Brenes A, Georgas J, Hui CH, Marin G, Setiadi B, Sinha JBP, Verma J, Spangenberg J, Touzard H, de Montmollin G. 1986. The measurement of the etic aspects of individualism and collectivism across cultures. Australian Journal of Psychology 38: 257-267.

Triandis HC, Bontempo R, Villareal MJ, Asai M, Lucca N. 1988. Individualism and collectivism: cross-cultural perspectives on self-ingroup relationships. Journal of Personality and Social Psychology 54: 323-338. 
Triandis HC, Chan DK-S, Bhawuk DPS, Iwao S, Sinha JBP. 1995. Multimethod probes of allocentrism and idiocentrism. International Journal of Psychology 30: 461-480.

Triandis HC, Gelfand MJ. 1998. Converging measurement of horizontal and vertical individualism and collectivism. Journal of Personality and Social Psychology 74: 118-128.

Triandis HC, Leung K, Villareal MJ, Clack FL. 1985. Allocentric versus idiocentric tendencies: convergent and discriminant validation. Journal of Research in Personality 19: 395-415.

Triandis HC, McCusker C, Betancourt H, Iwao S, Leung K, Salazar JM, Setiadi B, Sinha JBP, Touzard H, Zaleski Z. 1993. An etic-emic analysis of individualism and collectivism. Journal of Cross-Cultural Psychology 24: 366-383.

Triandis HC, McCusker C, Hui CH. 1990. Multimethod probes of individualism and collectivism. Journal of Personality and Social Psychology 59: 1006-1020.

Van Mechelen I, Kiers HAL. 1999. Individual differences in anxiety responses to stressful situations: a three-mode component analysis model. European Journal of Personality 13: 409-428.

Waterman AS. 1981. Individualism and interdependence. American Psychologist 36: 762-773.

Waterman AS. 1984. The Psychology of Individualism. Praeger: New York. 